\title{
Constrictive pericarditis and pleuropulmonary fibrosis secondary to cabergoline treatment for Parkinson's disease
}

\author{
M Townsend, D H Maclver
}

Heart 2004;90:e47 (http://www.heartjnl.com/cgi/content/full/90/8/e47). doi: 10.1136/hrt.2004.036236

\begin{abstract}
A 63 year old man with a six year history of Parkinson's disease presented with signs of right heart failure following a knee replacement. Constrictive pericarditis was diagnosed and a radical pericardectomy performed. Six months later, the patient remained unwell with raised inflammatory markers. An inflammatory fibrotic reaction caused by cabergoline was diagnosed. He improved after cessation of cabergoline.
\end{abstract}

t is important to recognise that commonly used drugs such as ergot alkaloids can cause progressive fibrosis with constrictive pericarditis. Examples of such drugs are bromocriptine, pergolide, cabergoline, lisuride, and the antimigraine drug methysergide. These drugs are widely used for the treatment of various conditions including Parkinson's disease. Cabergoline is a selective dopamine agonist with a greater affinity than that of bromocriptine. We describe a case of pleuropulmonary fibrosis and constrictive pericarditis caused by cabergoline.

\section{CASE REPORT}

A 63 year old man presented with a three month history of increasing bilateral leg swelling and exertional dyspnoea. His symptoms began a few weeks after a right total knee replacement. Parkinson's disease had been diagnosed six years previously and treated with co-careldopa 15.5/50 mg one tablet thrice daily, trihexyphenidyl $2 \mathrm{mg}$ daily, and cabergoline $5 \mathrm{mg}$ daily for three years. There was no other significant medical history.

Examination showed that he was in sinus rhythm with a pulse of 60 beats/min and blood pressure $110 / 60 \mathrm{~mm} \mathrm{Hg}$. His jugular venous pressure was increased at $18 \mathrm{~cm}$ with rapid $\mathrm{x}$ and y descents. On auscultation he had a soft ejection systolic murmur, loud pulmonary component of the second heart sound, and a loud third heart sound at the lower left sternal edge. His chest was clear, he had mild hepatomegaly, and there was pitting oedema of both legs. Table 1 shows his initial blood analysis results.

The provisional diagnosis was of a pulmonary embolism with pulmonary hypertension and right ventricular failure. The ECG showed $\mathrm{T}$ wave inversion in the precordial and inferior chest leads. The patient was admitted for administration of antithrombotic treatment and further evaluation.

A transthoracic echocardiogram showed a normal left ventricular ejection fraction. The right ventricle was mildly dilated with reasonable function and the estimated right ventricular systolic pressure was $36 \mathrm{~mm} \mathrm{Hg}$. Computed tomography (CT) of the chest was performed with contrast, which showed a right pleural effusion, a dilated inferior vena cava, and no pulmonary artery filling defects. A ventilationperfusion scan showed a single mismatched perfusion defect in the right lower lobe giving an intermediate probability of a pulmonary embolus.

The patient underwent cardiac catheterisation, which showed normal left ventricular function and normal coronary arteries. Pressure tracings showed increased and identical filling pressures with a dip and plateau pattern consistent with constrictive pericarditis. Pulmonary angiogram showed a small filling defect in one of the right inferior pulmonary arteries. The CT of the chest was reviewed and the pericardium was felt to be thickened reaching a maximum of $4 \mathrm{~mm}$.

The patient was anticoagulated and referred for pericardectomy. At operation constrictive pericarditis was confirmed, a radical pericardial excision was performed, and a right pleural effusion was drained. The histological appearance of the pericardium confirmed florid chronic inflammation.

The patient made a satisfactory early postoperative recovery. Six months later he felt non-specifically unwell and blood results showed raised inflammatory markers (table 1). A further echocardiogram was normal and a chest radiography showed pleural shadowing at the right base. Repeat chest CT showed new parenchymal scarring, pleural thickening, and calcification. Respiratory function tests showed a reduction in diffusion capacity and forced vital capacity, with a restrictive pattern consistent with nonspecific parenchymal lung disease.

The association between ergot alkaloids and fibrotic reactions was then recognised (table 2). Cabergoline was discontinued, after which ropinirole and oral steroids were commenced. Four months later he reported some modest improvement.

Table 1 Blood analysis results

\begin{tabular}{lll}
\hline Test & Initial result & Follow up \\
\hline Haemoglobin (g/dl) & 12.5 & 12.7 \\
White blood count $\left(\times 10^{9} / \mathrm{I}\right)$ & 5.5 & 6.95 \\
Platelets $\left(\times 10^{9} / \mathrm{l}\right)$ & 260 & 391 \\
ESR (mm in first hour) & - & 82 \\
C reactive protein (mg/l) & - & 36 \\
Plasma viscosity (cPa) & 1.77 & 1.93 \\
TSH (mU/l) & 2.67 & $\mathrm{ND}$ \\
Glucose (mmol/I) & 4.1 & $\mathrm{ND}$ \\
Sodium (mmol/I) & 141 & 138 \\
Potassium (mmol/I) & 4.1 & 4.5 \\
Urea (mmol/I) & 9.6 & 7.0 \\
Creatinine ( $\mu$ mol/I) & 116 & 91 \\
Bilirubin ( $\mu$ mol/I) & 14 & 10 \\
Alkaline phosphatase (U/I) & 344 & 264 \\
Alanine aminotransferase (U/I) & 23 & 20 \\
$\gamma$ Glutamyltransferase (U/I) & 240 & 153 \\
Albumin (g/l) & 39 & 34 \\
Calcium (mmol/l) & 2.26 & $\mathrm{ND}$ \\
Autoimmune profile & Negative & $\mathrm{ND}$ \\
\hline
\end{tabular}

ESR, erythrocyte sedimentation rate; ND, not done; TSH, thyroid stimulating hormone. 
Table 2 Number of yellow card reports received for fibrotic reactions with dopamine receptor antagonists

\begin{tabular}{lccl}
\hline Reaction & Pergolide & Bromocriptine & Cabergoline \\
\hline Pulmonary fibrosis & 8 & 6 & 1 \\
Pleural fibrosis & 8 & 4 & 2 \\
Pleural effusion & 13 & 4 & 3 \\
Fibrosing alveolitis & 2 & 0 & 0 \\
Retroperitoneal fibrosis & 12 & 5 & 0 \\
Constrictive pericarditis & 5 & 4 & 0 \\
Pericardial effusion & 2 & 1 & 0 \\
Total fibrotic reactions & 49 & 24 & 6 \\
\hline & & & \\
\hline
\end{tabular}

\section{DISCUSSION}

Our case illustrates the important association of constrictive pericarditis and drug induced fibrotic reactions. Recognised causes of pericardial constriction include viruses, tuberculosis, malignancy, uraemia, radiotherapy, and following cardiac surgery. Rarely, drugs such as hydralazine, practolol, procainamide, and methysergide have been implicated. ${ }^{1}$ More recently an association between newer ergot alkaloids and constrictive pericarditis has been recognised (table 2 ). ${ }^{2}{ }^{3}$

Cabergoline is an ergot alkaloid with longlasting and highly selective dopamine agonist activity. ${ }^{4}$ It has been shown to bind to the D2 receptor subtype with an affinity seven times that of bromocriptine. ${ }^{5}$ It is often used to treat hyperprolactinaemia, acromegaly, Nelson's syndrome, Cushing's disease, and Parkinson's disease. Other drugs with an ergoline derivative are bromocriptine, pergolide, lisuride, and the antimigraine drug methysergide.

This case report highlights the important association between ergot alkaloids and fibrotic reactions. To our knowledge, this is only the second recorded case of constrictive pericarditis and pleuropulmonary disease caused by cabergoline. ${ }^{6}$ However, owing to the non-specific, yet common, clinical findings constrictive pericarditis is likely to be under-recognised. Cabergoline has been shown to stimulate inflammatory reactions, and fibrosis appears to be associated with the drug's long term use. ${ }^{7}$ The pathogenesis of the fibrotic reaction is not understood. High dose corticosteroids have been used but it is unknown whether they alter outcome.

Medicine Control Agency data $^{8}$ suggest that before commencing treatment with an ergot derivative it may be appropriate to carry out baseline investigations including erythrocyte sedimentation rate, urea and electrolytes, chest radiography, and lung function tests. In view of the insidious onset of fibrotic disorders regular monitoring is recommended.

In summary, we present a case of constrictive pericarditis secondary to cabergoline treatment. Multiple drug regimens are increasingly frequent and we suggest that particular vigilance is needed in patients taking ergot derivatives. Increased inflammatory markers should raise the possibility of a fibrotic reaction. With increased awareness, we believe that this association may be found to be significantly more common than previously recognised.

\section{Authors' affiliations}

M Townsend, D H Maclver, Taunton and Somerset Hospital, Musgrove Park, Taunton, UK

Correspondence to: Dr Mandie Townsend, Department of Cardiology, Taunton and Somerset Hospital, Musgrove Park, Taunton TA1 5DA, UK; mandietownsend@yahoo.co.uk

Accepted 17 March 2004

\section{REFERENCES}

1 Braunwald E, Zipes D, Libby P. Heart disease: a textbook of cardiovascular disease, 6th ed. Philadelphia: WB Saunders, 2001.

2 Anon. Fibrotic reactions with pergolide and other ergot-derived dopamine receptor agonists. Curr Probl Pharmacovigilance 2002;28:3.

3 Frans E, Dom R, Demedts M. Pleuropulmonary changes during treatment of Parkinson's disease with a long-acting ergot derivative, cabergoline. Eur Respir J 1992;5:263-5.

4 Colao A, Lombardi G, Annunziato L. Cabergoline. Expert Opin Pharmacother 2000;1:555-74.

5 Ichikawa K, Kojima M. Pharmacological effects of cabergoline against Parkinson's disease. Nippon Yakurigaku Zasshi 2001;117:395-400.

6 Ling LH, Ahlskog JE, Munger TM, et al. Constrictive pericarditis and pleuropulmonary disease linked to ergot dopamine agonist therapy (cabergoline) for Parkinson's disease. Mayo Clin Proc 1999:74:371-5.

7 Frank W, Moritz R, Becke B, et al. Low dose cabergoline induced interstitial pneumonitis. Eur Respir J 1999;14:968-70.

8 Medicine Control Agency. Committee on Safety of Medicines. Current Problems in Pharmacovigilance 2002;28. 